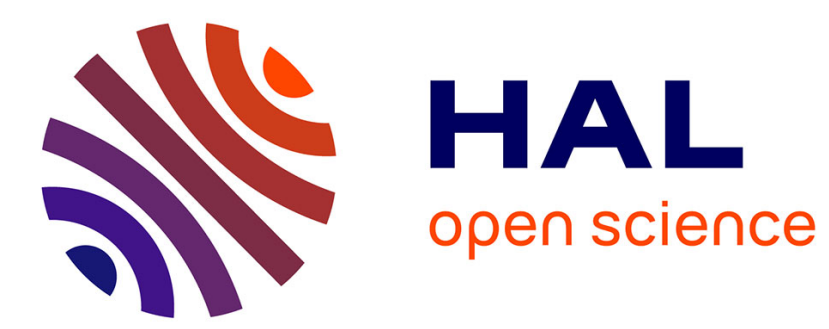

\title{
Un dîner prud'hommique à Lyon, avril 1856
}

Ludovic Frobert

\section{To cite this version:}

Ludovic Frobert. Un dîner prud'hommique à Lyon, avril 1856. Descendre, Romain; Fournel, JeanLouis. Langages, politique, histoire: avec Jean-Claude Zancarini, ENS Éditions, pp.539-550, 2015, Hors collection. halshs-01247570

\section{HAL Id: halshs-01247570 https://shs.hal.science/halshs-01247570}

Submitted on 19 Jun 2017

HAL is a multi-disciplinary open access archive for the deposit and dissemination of scientific research documents, whether they are published or not. The documents may come from teaching and research institutions in France or abroad, or from public or private research centers.
L'archive ouverte pluridisciplinaire HAL, est destinée au dépôt et à la diffusion de documents scientifiques de niveau recherche, publiés ou non, émanant des établissements d'enseignement et de recherche français ou étrangers, des laboratoires publics ou privés. 


\title{
Un diner prud'hommique à Lyon, avril 1856
}

\author{
Ludovic Frobert \\ Directeur de recherche CNRS/TRIANGLE
}

Le projet sur la petite presse ouvrière lyonnaise des années d'insurrection 1831-1834 et sur le mouvement social dans le milieu de la soierie, donc chez les célèbres canuts, a coïncidé avec les premières années du laboratoire TRIANGLE. Entre 2004 et 2010 il allait donner lieu à l'établissement d'un site d'édition critique des principaux journaux, dont le pionnier L'Echo de la fabrique ${ }^{l}$, à un colloque et un recueil ${ }^{2}$, à une thèse de doctorat ${ }^{3}$ et à diverses contributions individuelles ${ }^{4}$. Or tout ce projet est parti d'une discussion avec JeanClaude Zancarini courant 2003 dans les couloirs de l'Ecole Normale Supérieure Lettres et Sciences Humaines. Alors que l'ENS venait s'implanter à Lyon, Maurice Tournier avait signalé à Jean-Claude l'importance du corpus de la petite presse ouvrière et canuse, souligné les enjeux d'une redécouverte de ces journaux pionniers; et Jean-Claude, qui savait que je commençais à m'intéresser à cette période du premier $19^{\mathrm{e}}$ siècle, à ses turbulences et à ses réformateurs sociaux saint-simoniens, fouriéristes, républicains, communistes m'avait conseillé d'aller jeter un œil sur L'Echo de la fabrique. Dans le journal des tisseurs on trouvait de savoureux «coups de navette » : «Un prince a dit : «les intérêts des uns doivent être les intérêts des autres ». Les ouvriers sont toujours les uns, quand seront-ils les autres ? ${ }^{5}$, mais on trouvait aussi plus généralement une réflexion menée principalement par ces artisans sur les valeurs et les institutions d'une démocratie d'ateliers et, au-delà, d'une démocratie tout court.

Depuis trois ans, le travail sur la Fabrique et les canuts s'est poursuivi, mais à une échelle différente. Avec George Sheridan nous avons travaillé sur le très riche fonds d'archives du chef d'atelier en soierie Pierre Charnier ${ }^{6}$. Né en 1795, décédé en 1857, Charnier était un tisseur lyonnais, un chef d'atelier en soierie, un canut. Il fut l'un des principaux « chefs ouvriers » (Fernand Rude) de la cruciale période 1830-1848 bien qu'il se définissait

\footnotetext{
${ }^{1}$ http://echo-fabrique.ens-lyon.fr

${ }^{2}$ L. Frobert (dir.), L'Echo de la fabrique. Naissance de la presse ouvrière à Lyon (1831-1834), Lyon, ENSEditions, collection « Métamorphose du livre », 2010.

${ }^{3} \mathrm{~S}$. Hupfel, L'évolution comparée des manufactures de soieries de Lyon et de Londres, 1789-1848. Une approche institutionnaliste, Thèse Sciences économiques, ENS-Lyon, 2010. Cette Thèse a reçu le Prix du jeune chercheur de la Ville de Lyon 2011.

${ }^{4}$ Dont notamment, L. Frobert, Les Canuts, ou La démocratie turbulente (Lyon 1831-1834), Paris, Tallandier, 2009 ; S. Hupfel, "The Spitalfields Acts and the Classics : Ricardo, J.S. Mill, Bowring and Senior on the London Silk Industry (1823 to 1841)", The European Journal of the History of Economic Thought, 19(1), 2012,

${ }^{5} L^{\prime}$ Echo de la fabrique, $\mathrm{n}^{\circ} \mathrm{du} 22$ janvier 1832, p. 8.

${ }^{6}$ L. Frobert et G. Sheridan, Le Solitaire du ravin. Pierre Charnier (1795-1857), prud'homme tisseur et canut de Lyon, Lyon, ENS-Editions, collection « Le gouvernement en question », 2014.
} 
lui-même comme «un humble ciron de l'armée du progrès $»^{7}$; de fait, il se voyait comme une Lumière du peuple canut, une Lumière instruite tant par les gestes de l'atelier que par sa longue mission de représentant des tisseurs aux prud'hommes, ou encore par ses engagements politiques et religieux dans son quartier, enfin par ses lectures, ses promenades et ses enquêtes. Auteur vers 1827 de la toute première esquisse du mutuellisme des tisseurs de Lyon, Charnier fut impliqué quatre ans plus tard dans les évènements de l'automne 1831, envoyé comme représentant des ouvriers de Lyon pour plaider leur cause devant Casimir Périer ; en 1834 il se déplaça encore à Paris pour tenter de témoigner devant la Cour des Pair, qui allait le censurer, des exactions commises par les soldats du «Roi bourgeois» sur les tisseurs de Vaise et leurs familles. Charnier s'affirma surtout par la suite comme prud'homme chef d'atelier, constamment réélu à partir de 1832 et jusqu'à 1857 : un « magistrat plébéien élu par des prolétaires ${ }^{8}$, comme il aimait qu'on le définisse. Il œuvra alors en faveur des régulations démocratiques de la Fabrique, l'industrie de la soie lyonnaise, organisée sur le modèle complexe de la manufacture dispersée : "La Fabrique de soierie de la ville de Lyon, expliquait-il, est semblable à un très vaste et unique jardin ayant inépuisablement d'eau pour suffire à toutes sortes de besoins, lequel jardin divisé en portions, aurait pour l'exploitation de ces mêmes portions autant de jardiniers libres que de plantes ou de fleurs ${ }^{9}$.

Découverts par Fernand Rude au début des années 1930 et déposés aujourd'hui à la Bibliothèque Municipale de Lyon, les papiers Charnier sont d'une très grande richesse pour l'historien ${ }^{10}$. Bien sûr, ils offrent, en nombre, des renseignements cruciaux sur la vie de la Fabrique, sur ses institutions, les prud'hommes, les caisses de prêt, le mutuellisme, mais aussi les journaux des canuts, notamment le premier d'entre eux, L'Echo de la fabrique. Les «évènements », les «troubles de Lyon » (les «attentats de Lyon » vont avancer certaines autorités), ceux de novembre 1831 et d'avril 1834, ceux du printemps 1849, bénéficient également, grâce à ces archives, d'éclairages nouveaux. On apprend aussi, à la lecture de lettres, notes, témoignages, ce que fut au quotidien la vie, les affections et les missions de ce représentant canut (en tant que prud'homme), au sein de sa famille, dans son atelier et, audelà dans sa rue ou son quartier. On découvre un caractère, un tempérament. A un collègue, Délestra qui s'excuse de s'être emporté et d'avoir été colérique en présence du prud'homme, Charnier répond, "Croyez-vous que je sois exempt de votre infirmité ?... un homme tel que vous, Monsieur, ne doit pas ignorer qu'il y a des colères de diverses espèces, vous savez que celle qui est prompte à faire explosion ressemble à l'éclair qui disparaît soudainement; tel est le caractère de celle dont je suis affligé ${ }^{11}$. Enfin en parcourant ces documents originaux, on est confronté à une trajectoire singulière, assez improbable et non exempte de bifurcations, donc fascinante. Charnier signait ses interventions dans la presse, Le Solitaire du Ravin. Le ravin, c'était le quartier Saint-Paul où il habitait montée Saint-Barthélémy, sur la rive ouest de la Saône, lieu ancien de la canuserie, un quartier que venaient concurrencer désormais les pentes de la Croix-Rousse où s'installaient les nouvelles générations de tisseurs depuis la diffusion du métier Jacquard. Le ravin s'était aussi le lieu qui se situait exactement entre les

\footnotetext{
${ }^{7}$ Papiers Charnier, BML, boite 376, feuillets 295-304: «Journées d'avril/ Mémoire de Charnier sur son audition », Lyon, sans date.

${ }^{8}$ Papiers Charnier, BML, boite 379, feuillets 306-307 : Delestra à Charnier, Lyon, sans date, avec copie de la réponse de Charnier : Charnier à Mr Delestra, tisseur de soie, rue Juiverie, Lyon, le 5 février 1836.

${ }^{9}$ Papiers Charnier, BML, boite 376, feuillet 13 : Règlement de la Société de bienfaisance en indication et secours mutuels des chefs d'ateliers, de la fabrique de soierie de la ville et faubourgs de Lyon, Lyon, sans date.

${ }^{10}$ Fonds Fernand Rude, archives de Pierre Charnier, Bibliothèque municipale de Lyon, boites 375 à 380 .

${ }^{11}$ Document cité, échange Delestra/Charnier, boite 379, feuillets 306-307.
} 
deux collines qui se faisaient face et s'affrontaient, l'une, Fourvière, où on priait, l'autre, la Croix-Rousse, où on travaillait ; le ravin était pour cela un lieu indépendant, propre à penser et réaliser la réconciliation des deux collines. Solitaire, Charnier l'était de part sa doctrine, un curieux légitimisme rouge, qui prétendait articuler le principe de l'hérédité royale et celui du suffrage universel, et qui enjoignait d'expérimenter la démocratie à des niveaux intermédiaires, localisés, de la société et sur des terrains économiques. Le thème de la conciliation, emprunté tant à la pensée religieuse qu'à la pensée républicaine, sera d'ailleurs au cœur de sa réflexion et de son action notamment, bien sûr, aux prud'hommes. Lorsqu'au printemps 1848, figure ouvrière reconnue à Lyon, Charnier fut sollicité à la fois par la liste légitimiste et par celle des républicains modérés du quai de Retz il refusa, arguant qu'il voulait « rester prud'homme ».

Dans les archives on découvre donc Pierre Charnier, légitimiste et catholique depuis toujours, instruit, un temps, de façon critique, par le message saint-simonien, toujours attentif aux mots d'ordre républicains, protecteur inconditionnel de l'économie d'ateliers et de ses canuts pour lesquels il exige toujours plus de dialogues, de participations et de négociations, membre un temps de la Société de Saint-François Xavier au milieu des années 1840, défenseur encore de plusieurs Voraces en 1849 devant le Conseil de guerre appelé à juger les insurgés lyonnais de mai, mais ayant voté au printemps précédent pour la liste blanche. Ce que Charnier vomissait, c'était la France Louis-philipparde soumise aux sirènes de Guizot et de Thiers ( enrichissez-vous»), une France inégalitaire qui se mettait en place, celle des notables, celle qui fondée par l'insurrection de Juillet 1830 allait consacrer la violence de l'argent sous couvert de rhétorique libérale faisant ainsi disparaître les deux sources de la morale adaptée aux nouveaux temps industriels, la religion et la démocratie : "c'est au cri de liberté que vous trompiez, que vous égariez le peuple jusqu'au rôle de machine insurrectionnelle, interpellait-il les notables de 1830, afin d'en faire plus tard vos serfs, vos ilotes, vraie machine industrielle, fonctionnant par l'impulsion de votre liberté industriel qui est l'esclavage de l'artisan $\gg^{12}$.

Cette rhétorique libérale, pure idéologie à la solde des élites de Juillet puis à celle de l'Empire, avait été active y compris donc dans le monde de la Fabrique et des soieries de Lyon ; elle était, de fait, liberticide pour le monde des artisans Ce discours avait en effet incessamment souligné la supériorité du modèle hiérarchique et fonctionnel de la manufacture concentrée (la laine et le coton) sur celui de la Fabrique, de ses artisans autonomes et de ses règlements byzantins régissant des dizaines de petits ateliers dispersés sur la colline de la Croix-Rousse et dans les vieux quartiers de la rive ouest de la Saône ; des règlements en constantes évolution, continûment négociés dans le cadre des prud'hommes entre partenaires industriels, négociants et chefs d'ateliers. Depuis 1806, dans l'idéal lieu du paritarisme et de la négociation, temple espéré de la conciliation, les prud'hommes, notamment dans la section de la Fabrique (l'industrie de la soie) étaient l'enjeu de conflits et constantes réorganisations dépendantes de l'évolution des rapports de force, et de la conjoncture politique et sociale. Les chefs d'ateliers et leurs représentants exigeaient que l'institution assume sa logique paritaire et devienne un vrai lieu de négociation permettant de contrôler les abus incessant créés par l'asymétrie de pouvoir entre le négociant, donneur d'ordre, et le chef d'atelier. C'est dans le même esprit que les tisseurs s'étaient associés dès la fin des années 1820 , créant le Devoir Mutuel, premier mutuellisme des tisseurs où il était question, avant tout, de «s'indiquer » et de faire collectivement poids. Une fraction des négociants allait être constamment sensible à cette revendication, pour des question de doctrine et de concession aux idées nouvelles (saint-

\footnotetext{
${ }^{12}$ Papiers Charnier, BML, boite 378, feuillet 93: Protestations diverses contre la vente des biens de LouisPhilippe, suivies de commentaires de Pierre Charnier, Lyon, le 29 janvier 1852.
} 
simoniennes, fouriéristes, républicaines), mais aussi pour des questions d'intérêt ; car le bon fonctionnement des prud'hommes, en tant qu'institution de régulation intermédiaire, constituait aux yeux de ces négociants un gage d'efficacité dans une industrie où l'innovation constituait un enjeu vital. Mais au gré des évènements, une fraction importante des négociants allait être hostile à ce que représentaient les prud'hommes, ils allaient défendre une conception minimale de l'institution et s'accrocher à tous les règlements, pousser les avantages, pouvant accentuer les déséquilibres entre représentants canuts et représentants négociants ; bref à vider l'institution de sa logique de participation et de paritarisme. Charnier fut de 1832 à 1857 au cœur de ces arrangements et de ces affrontements fluctuants qui firent la vie des prud'hommes. Il se confronta à ces réalités lors de ses différentes expériences en tant que prud'homme chef d'atelier: au sein même des prud'hommes lors des séances hebdomadaires, mais aussi hors des prud'hommes lors des très importantes missions de surveillance des ateliers. Il devint un expert en matières de causes mettant en question les piquages d'once, intervint dans des arbitrages relatifs aux techniques et aux métiers à tisser, et fut surtout accaparé par la question de l'apprentissage. Il fut un historien et un doctrinaire de l'institution prud'hommale : un historien rassemblant et consultant les anciens règlements datant du $18^{\mathrm{e}}$ siècle et qui avaient réglementés les relations entre maîtres-ouvriers et maîtresgardes aux temps, déjà, des émeutes de 1744 et 1786 ; un doctrinaire qui allait s'engager dès 1832 dans certaines des polémiques saillantes des nouveaux prud'hommes, qu'elles concernent la libre-défense ou la jurisprudence fixe. En juin 1856 son ami l'abbé Combet lui faisait parvenir, non sans facétie, un petit poème :
Monsieur Charnier,
De tous prud'hommes excellents
Qu'on vante le plus en ces temps,
Charnier, aucun ne vous ressemble ;
Leur art cède à votre secret ;
Car vous devenez tout ensemble,
Chef, ouvrier et juge parfait ${ }^{13}$.

Charnier se voyait donc, avant tout comme un "prud'homme tisseur», un représentant, un fonctionnaire public du peuple canut. Il remplit sa mission dans des conjonctures politiques et sociales plus ou moins favorable. Et dans les contextes de tension, il se plut à jouer le rôle de « ciron », c'est-à-dire le rôle d'un parasite, dérangeant, irritant les pouvoirs en place. Le début des années 1850 fut caractéristique d'une telle période. En dépit du caractère très décalée de son idéologie, un légitimisme rouge, Charnier qui avait été un temps mis à l'écart, suspectés par les uns pour ses options républicaines, stigmatisés par les autres pour son catholicisme, Charnier était revenu en pleine lumière grâce à l'inattendue défense victorieuse qu'il avait assuré d'une dizaine de mutuellistes et de Voraces trainés devant le Conseil de guerre à l'automne 1849. Il était devenu un sage, un sage toujours aussi dérangeant pour ses adversaires. Au début de l'Empire, et de ses nouvelles velléités de libéralisme autoritaire, l'institution des prud'hommes était de nouveau en procès. Aux prud'hommes de Lyon, et pour la section de la Fabrique, le président (toujours choisit chez les négociants par ailleurs majoritaires) était Félix Bertrand, un homme qui ne cachait pas sa morgue vis-à-vis des représentants canuts. Charnier connaissait Bertrand de longue date. En 1845, il l'avait déjà affronté alors que le prud'homme négociant, informant un jurisconsulte allemand sur la logique des prud'hommes, s'était «distingué » en expliquant au visiteur que « chacun de nous défend les intérêts de sa caste ». Charnier l'avait alors vertement repris : « je

\footnotetext{
${ }^{13}$ Papiers Charnier, BML, boite 376, feuillets 378 : Charnier à Mon cher confrère, Lyon, le 30 novembre 1845.
} 


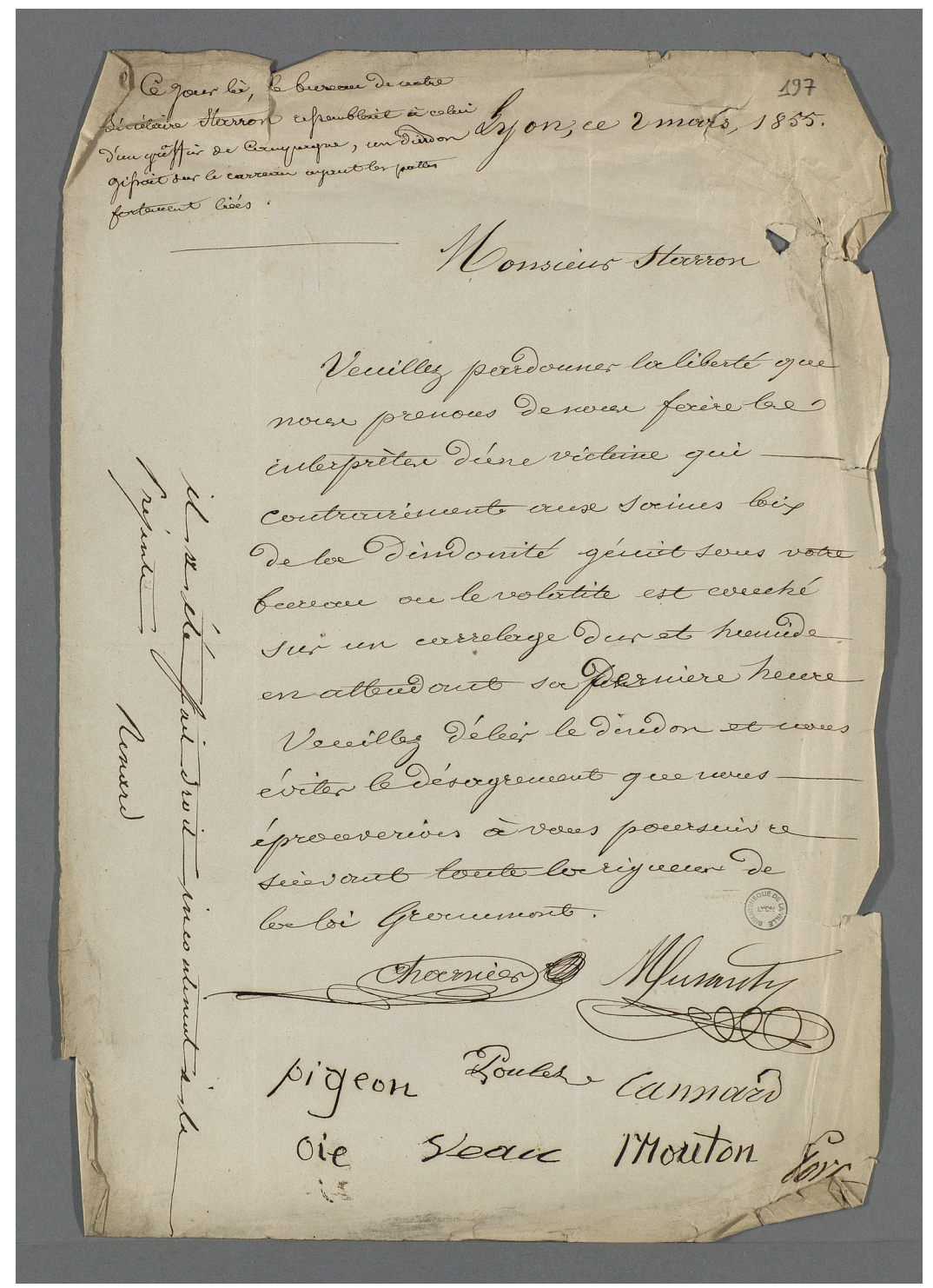

vous considère donc comme ayant porté atteinte non seulement à votre propre considération mais à celle de vos collègues, maîtrefabricants et chefs d'ateliers, par un portrait tout à fait défiguré et rabougri sur l'esprit du conseil » ${ }^{14}$. Devenu président des prud'hommes pour la section de la Fabrique en 1852, Bertrand avait maintenu une ligne similaire, et ces années furent marquées par de constantes escarmouches entre lui et Charnier. Ces escarmouches prenaient parfois des formes inattendues. En voilà un exemple: Le président des prud'homme avait toujours comme homme-lige un secrétaire, et le secrétaire de Bertrand était alors le dénommé Starron, créatures insignifiante et veule aux yeux de Charnier. En mars 1855 , entrant dans la salle du conseil pour une séance hebdomadaire, Charnier et son collègue représentant tisseur Muzanty eurent la surprise de constater qu'au pied du bureau du sieur Starron gisait un volatile ligoté. Staron confondait-il donc le conseil des prud'hommes avec un aimable tribunal de campagne? L'épisode conduisit les deux représentants-tisseurs à plaisanter le sieur Starron en lui transmettant la missive suivante cosignée Charnier, Muzanty mais aussi PIGEON, POULET, CANARD, OIE, VEAU, MOUTON, PORC et enfin RENARD :

\section{Monsieur Starron,}

Veuillez pardonner la liberté que nous prenons de nous faire les interprètes d'une victime qui, contrairement aux saines lois de la dindonité gémit sous votre bureau où le volatile est couché sur un carrelage dur et humide en attendant sa dernière heure.

Veuillez délier le dindon et nous éviter le désagrément que nous éprouverions à vous poursuivre suivant toute la rigueur de la loi Grammont ${ }^{15}$.

\footnotetext{
${ }^{14}$ Papiers Charnier, BML, boite 375, feuillets 31-32: Lettre de Pierre Charnier à Bertrand, conseiller prud'homme, au sujet d'une querelle sur les positions adoptées lors d'un Conseil des Prud'hommes, Lyon, le 17 juin 1845 .
}

\footnotetext{
${ }^{15}$ Papiers Charnier, BML, boite 375, feuillet 197: Lettre adressée à Starron, secrétaire du Conseil des
} 
Le document que nous éditons ci-après ce situe dans une même perspective. Il s'agit du récit d'un «diner prud'hommique» auquel assista Charnier en avril 1856 et d'une première rencontre avec la «gastronomie bourgeoise ». Le témoignage est remarquable et apporte de nombreux éléments d'information sur la forme que pouvait prendre la contestation plus ou moins goguenarde chez le Solitaire du ravin et sur la façon dont les autres représentants, principalement mais pas seulement négociants, tentaient d'esquiver les piques du vieux ciron. Le récit permet également de saisir sur cas la confrontation, en matière de convivialité, de commensalité et d'alimentation, entre l'univers des tisseurs, plus attirés par la table de Guignol et Gnafron, et celui des négociants, ces derniers alors friands d'innovations gastronomique et de cénacles de gastrolâtres ${ }^{16}$. Il renseigne aussi en filigrane sur les rapports de pouvoir au sein de la confrérie des prud'hommes, une confrérie où les représentants étaient égaux mais où certains l'étaient bien plus que d'autres et où les négociants mettaient constamment, matériellement et symboliquement, les chefs d'ateliers sur le reculoir. Enfin, de façon plus fine encore, ce récit permet de saisir des effets de génération et la fragmentation ou les tensions qui pouvaient apparaître au sein même du groupe des représentants ouvriers. Car Charnier représentait alors une ancienne canuserie, pétrie de valeurs morales, indissociablement civiles et religieuses, une canuserie éduquée et instruite, attentive à l'apprentissage de la conciliation ; une canuserie soucieuse de la composition du passé et de l'avenir et qui voyait surgir depuis les années 1840 et notamment depuis 1848 une jeune représentation ouvrière moins strictement liée à l'habitus de la Fabrique, pressée de confondre la question industrielle, le travail, avec une question politique synonyme d'insurrection, plus encline, au final, à céder aux charmes de la ploutocratie.

« diner prud'hommique à l'hôtel Bocquis » ${ }^{17}$

Rendez-vous des 9 tisseurs au tour du Cheval de bronze.

Entrée à $5 \mathrm{~h} 10 \mathrm{mn}$.

Le président s'est approché de moi d'un air goguenard : Dites donc Mr Charnier, en attendant le diner, vous devriez faire une motion sur la disjonction ${ }^{18}$. Je me suis levé précipitamment en

Prud'hommes, signée de Pierre Charnier, d'un autre membre du Conseil (Muzanty), Lyon, le 2 mars 1855.

\footnotetext{
${ }^{16}$ Voir ici les contributions réunies dans MA. Privat-Savigny, Histoire de la gastronomie à Lyon, Milan, Silvana Editoriale, 2011 ; Egalement, J. Barou, «Rituels et fonctions des sociétés gastronomiques lyonnaises », Ethnologie française, 27 (1), 1997, pp. 9-17.
}

\footnotetext{
${ }^{17}$ Papiers Charnier, BML, boite 378, feuillets 263-265, Notes pour mémoire, $11^{\mathrm{e}}$ cahier.

${ }^{18}$ Note d'édition : Il s'agit là d'une petite provocation du président Bertrand à l'encontre de Charnier. Les deux hommes s'étaient affrontés peu avant aux prud'hommes sur la question de la disjonction. En 1854, le débat concernait l'organisation des délibérations lors des séances du conseil. Réfléchissant sur l'organisation de cette procédure et sur les moyens de l'améliorer, Charnier proposait « deux grandes audiences par semaine. L'une pour la fabrique d'étoffes et l'autre pour toutes les autres questions ». La façon dont sa proposition fut écartée par le président et par «ses compères intéressés à repousser la disjonction » heurta vivement Charnier. Bertrand arguait de l'indivisibilité du bureau général des prud'hommes; mais la vraie raison, constatait Charnier était que le «despotisme» de ce Président exprimait surtout la volonté des négociants de pérenniser les situations d'absence de parité au sein des prud'hommes : «puisque nous n'avons pas le droit de diviser le conseil en excluant une partie des membres du conseil, notait Charnier, pourquoi sur 9 prud'hommes tisseurs est ce qu'il n'y en a que 3 siégeant au bureau général ? Si cela est permis nous pouvons également diviser pour le prétendu bureau général ». Dans le cadre du diner prud'hommique, Charnier va prendre au mot Bertrand ce qui va créer l'embarras de son président et permettre à Charnier, en guise de mise en bouche, de mettre les pieds dans le plat.
} 
répondant : je me rend à votre invitation, et me plaçant au milieu du salon... Messieurs ! Je réclame votre attention un instant, je ne fais que me rendre à l'invitation de Mr le PrésidentOh! non répartit le Président avec le rouge au front, l'on votera sur la question avant d'entrer en matière - vous n'avez pas fait cette réserve. J'insiste et d'ailleurs je n'ai que deux mots à lire, il n'y a rien de mon cru l'on ne pourra pas supposer que c'est amour-propre de ma part. Je lu un extrait du Siècle, numéro du 16 mars dernier. Messieurs! Attention s'il vous plait: «Une seconde conséquence de la paix, c'est que l'attention du public et de la presse portera désormais plus complètement sur les questions de réforme intérieure, politiques ou économiques. Déjà, ce matin, le Journal des débats demande la révision de la législation sur les sucres ».

Mr Combes, l'un des six juges des 19 causes de chapellerie pendant l'année dernière répondit : ah! Voilà de la douceur.

Ce jeu de mot fut approuvé par la presque totalité de l'assemblée qui appréhendait de voir la question prise au sérieux. - Messieurs, si vous ne croyez pas la question digne d'être prise en considération, je ne partage pas votre avis. Aucun de mes collègues tisseurs n'ayant témoigné leur sentiment, je me suis résigné au silence.

Le diner est annoncé, servi.

Nous fûmes introduits dans une magnifique salle. Quel contraste avec mon taudis. La table était d'une élégance princière, un immense plateau entièrement jonché de fleurs figurait au milieu, les mets exquis que nous allions consommer apparaissaient sous des formes élégantes, quatre verres de formes et dimensions différentes annonçaient qu'on avait le choix de quatre qualités de vins.

Quel contraste avec ma table sans nappe !

La place qui m'est réservée est presque en face du Président et à gauche du vice-président, quel honneur! Disons plutôt quelle fine combinaison de lier les mécontents au faisceau des satisfaits.

L'on mange, l'on broute, on déguste faisans et bécasses, bien faisandées, véritable contagion de luxe ; à peine j'eu gouté cette chair poison que je m'écriai : oh ! quelle charogne ! Mon voisin de gauche, habitué au bon ton en a beaucoup ri et en continuant de savourer la putréfaction dit qu'il se rappellerait du mot.

Le champagne apparaît ! ah ! pour le coup voilà les toasts qui vont commencer. Le Président se lève, il pose le verre en main, son attitude est solennelle, il porte un toast à l'Empereur. Le vice-président, c'est-à-dire l'aspirant au fauteuil porte un toast à son protecteur né, le Président, dont il fait le panégyrique par l'énumération de ses hautes capacités. Le Président répliqua par un pareil coup d'encensage, peignant le vice-président comme son digne et prochain successeur. Bien entendu, il énuméra ses capacités, à ces mots, le vice-président baissa modestement les yeux, sa tête s'est légèrement inclinée exprimant une approbation reconnaissante. Viennent les toasts de pacotilles parmi lesquels celui de mon confrère Tray tisseur, à l'Empereur fondateur du conseil des prud'hommes en 1806.

J'ai pris la parole en ces termes : Messieurs! Je crois devoir éclaircir un fait historique, $\mathrm{Mr}$ Tray a cité le restaurateur du conseil des prud'hommes comme fondateur, tandis que c'est à Saint-Louis que nous devons cette institution.

Le dessert circule sous diverses formes, celles qui imitent la nature sont sans contredit les plus élégantes. Mr Favrot m'offre cordialement un tronçon de pêche avec le noyau en me faisant observer que vu l'extrême rareté de ce fruit dans cette saison, l'on n'en prend qu'un tronçon, j'acceptai gaiement, je me disposais à déguster avec attention pour comparer la différence qui existait entre la saveur du fruit conservé d'avec celle d'une même espèce cueilli récemment, mais, ô surprise ! c'était un petit chef-d'œuvre d'imitation. Honneur au confiseur ; honneur à qui de droit. 
J'ai soigneusement plié et empoché ce tronçon de pêche pour égayer ma femme et ma fille. J'y ai joint d'autres imitations, petites raves, noisettes, etc de cette manière, le diner princier a réjoui ma famille. Là c'était une joie pure comme tout contentement de peu.

Les toasts commencent à être étouffés par ce bruit des conversations particulières ; enfin le dessert est terminé par l'annonce du café, on passe au salon.

Les plus timides deviennent les plus loquaces, encouragés par le fumet des viandes arrosées du pétillant champagne. Ils se croient orateurs. Carbonnel, ex-président d'un club passablement démocratique entre ou plutôt veut entrer en digression sur les obligations des prud'hommes au travers d'une discordance de paroles baignantes de timidité et d'affectation. L'on ne comprend qu'un sens, c'est un compliment au digne Président qui a su se rallier le conseil. L'un des assistants riait de cette éloquence burlesque, il m'interrogea par un regard sardonique, je m'approchais de lui, alors il me demanda ce que j'en pensais de cette éloquence burlesque - c'est un ex-président d'un club démocratique qui jette sa blouse de février 1848 sur le buisson. Sa réplique fut claire, c'était un rire approbateur. Malheureusement une scène bien regrettable devait succéder à ce sincère et muet entretien. Ah! voilà le bouquet qui s'approche ! Rougement porte un toast! au Président! il répète ce qui s'est dit en d'autres termes, cependant il sent qu'il faut du nouveau, il dit : malgré que $\mathrm{Mr}$ le Président parfois prend un ton un peu... enfin, un ton rodomont - j'accepte, dit le Président, oui j'accepte l'épithète, cela est vrai et quand j'ai pris un ton rodomont c'est que je l'ai cru utile dans l'intérêt de la justice. Rougemont continue, mais l'attention des auditeurs faisant défaut, il a terminé en réitérant son toast au Président. La causerie principale entoure le Président, les flatteries les plus surannées l'assiègent, c'était la gloutonnerie reconnaissante. Je témoigne ma reconnaissance de ce que le Président ait prévu le cas de mort - Comment donc ? réplique ce dernier - C'est bien clair vous avez désigné le vice-président pour votre successeur, voilà la prévision de votre mort. Le Président resta court ; je le tirai d'embarras par ces mots : voyez Messieurs la physionomie de notre président, elle exprime ces paroles: ah! vous me croyez emplâtré pour longtemps. Je saurai bien me déplâtrer. Le Président me tendit les deux mains, il me serra avec effusion, avec approbation puis dit: vous dites vrai.

Une discussion s'engagea entre Rougemont et le Président, Rougemont s'avoue presque vaincu sur le terrain de la disjonction; Combes, l'hercule des 6 prud'hommes, juge de 13 causes par an, élu par 10 à 12 électeurs, l'adjoint de la mairie de la Guillotière (aujourd'hui $3^{\mathrm{e}}$ arrondissement) chargé des mœurs attaque brusquement la discussion ; son œil est en feu, ses robustes poignets de crispent et voici comment il confond Rougement: Vous êtes des blagueurs - Nous des blagueurs - oui vous êtes tous des blagueurs, qu'est ce que c'est que toute votre causerie? C'est une paillarderie ? ... ce n'est rien du tout. Alors le Président intervint en plaçant sa main sur l'épaule de l'hercule aux galettes de fonte - Combes vous avez tort. A ces mots Combes s'inclina respectueusement et tout fut fini. C'était temps ${ }^{19}$.

Malheureusement je n'ai pas été prodigue de vin, j'avais conservé tout mon état normal. Je pus apprécier la triste situation qui nous était faite par cette insolente et grossière qualification. Mon horreur pour le duel et la bataillarderie m'ont fait conserver mon calme. Ce qui me peinait c'était de me voir obligé à rester contre envers mon collègue Rougement qui par ses ondulations au Président s'était par trop révélé.

\footnotetext{
${ }^{19}$ Note d'édition : Dans ces derniers paragraphes qui concernent des prud'hommes tisseurs, tels Carbonnel ou Combes, Charnier règle ses comptes avec les ex-insurgés rouges de 1848 . Il note comment ces exaltés et autres sanguins de 1848 ont acceptés sans broncher quelques années plus tard tant les mors que les sangles de l'Empire et de ses élites. Carbonnel se prosterne devant Bertrand, alors que Combes, qui avait été membre du comité révolutionnaire occupant la mairie de la Croix-Rousse en juin 1848 (un Combe dont Charnier moque en outre la représentativité : «10 à 12 électeurs ») est devenu un simple pandore aux ordres de Bertrand lors de ce piteux échange avec Rougemont.
} 
Après cette scène de taverne je circulai parmi les causeurs pour annoncer qu'il était trop tard, enfin à $10 \mathrm{~h}$ nous sommes descendus de ce lieu où après avoir bu à la parfaite union du conseil, on faillit se prendre aux cheveux.

Oh ! Boileau que n'étais-tu présent !

L'on me demanda ce que je pensais de la rodomontade du Président ? Je n'ai pas le droit de blâme sur ce sujet, que celui qui est sans péché jette la première pierre, au résumé : nous devons parler en présence du Président comme en son absence. Le Président a applaudi à ce principe. 